\title{
The Performance Comparison of European DTTV Standards with LDPC-Encoded-DVB-T Standard under AWGN Channel
}

\author{
Oktay Karakuş \\ Department of Electrical and Electronics Engineering \\ Izmir Institute of Technology \\ Izmir, Turkey \\ oktaykarakus@iyte.edu.tr
}

\begin{abstract}
In this study, in addition to previous work which is a performance comparison of European DTTV Broadcasting standards which are known as DVB-T and DVB-T2, DVB-T2's inner encoder/interleaver block which has LDPC Encoder/Bit Interleaver, is integrated into DVB-T instead of its own inner encoder/interleaver block. This Proposed System's performance under AWGN channel is compared to the DVB-T/T2 standards. The Proposed System achieves better performance results according to DVB-T and very close results according to DVB-T2. It achieves nearly from 4 to 7 decibels SNR gain and up to $13 \mathrm{Mb} / \mathrm{s}$ data rate gain then DVB-T results according to different code rate and modulation parameters.
\end{abstract}

Keywords: European DTTV Standards, DVB-T, DVB-T2, AWGN Channel, LDPC Encoder, Performance Comparison.

\section{Introduction}

Depending on the rapid advancement of technology in recent years, significant improvements have been observed in television broadcasting standards. The current technologies allow broadcasting in High Definition (HD) and 3-Dimension (3D) and watching this content at fixed or mobile receivers. In addition to this, the development on every area of wireless communications affects analog terrestrial television broadcasting technologies, and gives rise to an idea of digital terrestrial television broadcasting. With these developments, since the beginning of the 90s, digital terrestrial television broadcasting has become an important study area in the whole world.

Digital Video Broadcasting (DVB) project started to prepare specifications about digital terrestrial television in Europe in 1993. DVB can be defined as an industry-led consortium of broadcasters, manufacturers, network operators, software developers and regularity bodies committed to designing open technical standards for digital television and data services [1]. DVB Organization has prepared and published digital transmission standards for Europe. These are; 
- DVB-S, for Satellite Systems [2],

- DVB-C, for Cable Systems [3],

- DVB-H, for Handheld Systems [4],

- DVB-T, for Terrestrial Systems [5].

Although DVB-T has many advantages and is a great success in digital broadcasting area, it requires some improvements. These improvements should be in the area of adaptation for portable devices that moves faster and that is inside of the buildings. These facts provide a new and updated standard for DVB-T and this led to the standardization of DVB-T2. DVB-T2 specification was firstly published in September 2009 and in this document, new baseline transmission system is described in [6].

In a previous work [7], both of the standards are simulated under the effects of Additive White Gaussian Noise (AWGN) Channel and the acquired results are compared according to the Target Bit Error Rate (BER) value which is stated in standards. The results showed that DVB-T2 standard outperforms DVB-T standard under AWGN Channel and achieves up to 7 decibels power gain according to code rate and modulation parameters.

Low Density Parity Check (LDPC) Codes usage may be most important advantage of DVB-T2 Systems. In this study, it's going to be searched an answer to the question, "What will be the performance results for DVB-T system with LDPC Encoder?"

In literature, a study [8] applied this kind of idea to the DVB-T. In that study, writer uses just 16QAM modulation technique and substitute LDPC block with RSConvolutional Coding block. In the present study, LDPC Coding is used instead of Convolutional Coding. RS Coding block is also applied after inner interleaving block and in addition to 16QAM, QPSK and 64QAM Modulation Schemes are also used in this study.

This paper is organized as follows. First of all, DVB-T/T2 system architectures are briefly explained in section 2 . In section 3 , the proposed system is explained. Also the performance comparison and results is given in section 4. Paper ends by concluding the study.

\section{DVB-T/T2 System Architectures}

Transmitter structures of DVB-T and DVB-T2 systems are defined and published by DVB organization. DVB-T and DVB-T2's system architectures are briefly explained in this section but deeply defined in [5] and [6] respectively.

Receiver structures of DVB systems have no any specifications but "Implementation Guidelines" [9], [10]. These documents give information about the main blocks of systems' receivers and different ways of implementation for some specific blocks.

System architecture for DVB-T and DVB-T2 is provided in this section and in addition theoretical comparison of both is illustrated in table 1 .

\subsection{DVB-T Transmitter System Architecture}

DVB-T systems are designed to perform adaptation of the baseband TV signals from the output of the MPEG-2 transport multiplexer to terrestrial channel characteristics. 